Pure Mathematical Sciences, Vol. 3, 2014, no. 3, 113 - 120

HIKARI Ltd, www.m-hikari.com

http://dx.doi.org/10.12988/pms.2014.4511

\title{
A Note on Extreme Sets for Quasi-Continuous Functions
}

\author{
A. Daghighi \\ Linköping University, SE-581 83, Sweden
}

Copyright (c) 2014 A. Daghighi. This is an open access article distributed under the Creative Commons Attribution License, which permits unrestricted use, distribution, and reproduction in any medium, provided the original work is properly cited.

\begin{abstract}
It is known that for $\Omega$ a connected separable metric space, a function $\Omega \rightarrow \mathbb{R}$ which at every point attains a weak local extremum must have countable image. Let $\Omega \subset \mathbb{R}^{n}$ be a bounded domain and $f: \Omega \rightarrow \mathbb{C}$ a measurable, bounded, quasi-continuous function. For $n \in \mathbb{Z}_{+}$, we show that if $|f|$ has countable image then $f$ attains weak local extremum at each point of a dense open subset. We show by examples the optimality of the result, in the sense that strengthening the countability condition to discreteness (or even finiteness) will not affect the dense open condition. Conversely it is easily verified that if $f$ attains weak local extremum off a countable set then $|f|$ has countable image.
\end{abstract}

Mathematics Subject Classification: 26A15, 54C08, 54C30

Keywords: Quasi-continuous functions, weak local extremum

\section{INTRODUCTION}

Recently Behrends et al. [1] gave a short proof of the fact that if $\Omega$ is a connected separable metric space, and if $f: \Omega \rightarrow \mathbb{R}$ attains weak local extremum at every point, then (the image) $f(\Omega)$ is countable. In this text we shall only consider bounded domains $\Omega$ in $\mathbb{R}^{n}$ (as opposed to the more general case of connected separable metric $\Omega$ ), and complex or real-valued functions. Therefore we shall use the following definition.

Definition 1.1 (Weak local extremum). Let $\Omega$ be a topological space and $f$ : $\Omega \rightarrow Y$ for a metric space $\left(Y,|\cdot|_{Y}\right)$. The function $f$ is said to attain a weak local maximum (minimum) at a point $p_{0} \in \Omega$ if there exists an open $U \subset \Omega$, $p_{0} \in U$, such that $|f(z)|_{Y} \leq\left|f\left(p_{0}\right)\right|_{Y}\left(|f(z)|_{Y} \geq\left|f\left(p_{0}\right)\right|_{Y}\right)$ for all $z \in U . f$ 
is said to have a weak local extremum at $p$ if it attains either a weak local maximum or a weak local minimum at $p$. In the special case that $Y=\mathbb{R}$ we replace $|f(\cdot)|_{Y}$ by $f(\cdot)$.

In particular $\Omega$ and the target space of our functions will always be second countable $^{1}$. The only continuous functions which attain weak local extremum at every point of $\Omega$ are the constant functions. This is no longer true for the case of quasi-continuous functions.

Definition 1.2 (See Kempisty [6]). A function $f: X \rightarrow Y$ from a topological space $X$ to a metric space $(Y, d)$ is called quasi-continuous at $p_{0} \in X$ if $\forall \epsilon>0$ and each neighborhood $\mathrm{U}_{p_{0}}$ of $p_{0}$ there exists a nonempty open set $\omega_{p_{0}} \subset \mathrm{U}_{p_{0}}$ such that $d\left(f(x), f\left(p_{0}\right)\right)<\epsilon, \forall x \in \omega_{p_{0}}$.

The first author recently proved the following characterization.

Proposition 1.3 (Daghighi [4], Proposition 3.15, p.670). Let $\Omega$ be an open subset of some $\mathbb{R}^{n}$ (or $\mathbb{C}^{n}$ ), $p_{0} \in \Omega$, and let $f$ be a measurable locally bounded function $\Omega \rightarrow K$ (where $K=\mathbb{R}$ or $\mathbb{C}$ ). Then the following assertions are equivalent:

(i) $f$ is quasi-continuous at $p_{0} \in \Omega$.

(ii) There exists an open set $U_{p_{0}} \subset \Omega$ with $p_{0} \in \bar{U}_{p_{0}}$, such that for any sequence $\left\{z_{j}\right\}_{j \in \mathbb{N}}$ in $U_{p_{0}}$ with $z_{j} \rightarrow p_{0}$, it holds true that $f\left(z_{j}\right) \rightarrow f\left(p_{0}\right)$.

It is not sufficient for a continuous real-valued function with weak local extrema (or even strict local maxima) which is dense, to reduce to a constant, see e.g. Posey \& Vaughan [10] and Villani [11] who proved that for any countable subset of a metric space there is a real-valued continuous functions whose set of strict local maxima contains the given set. In the latter case the set of extreme points will have empty interior, since each strict local maximum is the boundary of two one-sided open intervals whose images under the continuous function contains an interval. In this paper we shall be concerned with the following.

Question: How can conditions on the image of the absolute value be used to determine the sets of weak local extrema?

Of course a special case would be to inquire sufficient conditions on the image of the absolute value in order for a quasi-continuous function to attain weak local extremum at every point (we shall later see, however, that such conditions must be quite restrictive and therefore less interesting, which is why we will allow exceptional sets in our study). It is not sufficient, for reduction to a constant, for a quasi-continuous function with weak local extremum at every point, that the image be countable.

\footnotetext{
${ }^{1} \mathrm{~A}$ countable basis for the topology can be taken as the family of all products of intervals with rational ends.
} 
Example 1.4. Let $\Omega=(-1,1) \subset \mathbb{R}$ and define the function $f: \Omega \rightarrow \mathbb{R}$ as follows: $f(x):=\frac{k+1}{k}$, for $x \in\left[\frac{1}{k+1}, \frac{1}{k}\right)$, and $f(x):=\frac{k-1}{k}$, for $x \in\left(-\frac{1}{k},-\frac{1}{k+1}\right]$, $k \in \mathbb{Z}_{+}, f(0)=1$. Then $f(x)$ is quasi-continuous on $\Omega$. The image $f(\Omega)=$ $\{y \in \mathbb{R}: y=f(x)$, some $x \in \Omega\}$ is a countable set of points, namely $f(\Omega)=$ $\left\{1 \pm \frac{1}{k}: k \in \mathbb{Z} \backslash\{0\}\right\}$, and therefore,

$$
|f|(\Omega):=\left\{y \in \mathbb{R}_{\geq 0}: y=|f(x)|, \text { some } x \in \Omega\right\},
$$

is a countable subset of $\mathbb{R}_{\geq 0}$. It is clear that any open interval of $p_{0}=0$ in $\Omega$, must contain two different points $x_{1}, x_{2}$ such that $\left|f\left(x_{1}\right)\right|<\left|f\left(p_{0}\right)\right|<\left|f\left(x_{2}\right)\right|$. Hence $f$ does not attain a weak local extremum at $p_{0}$ (but this is also the only point of $\Omega$ at which $f$ does not attain a weak local extremum).

Obviously, there are plenty of examples of non-constant quasi-continuous functions which attain weak local extremum everywhere.

Example 1.5. Let $\Omega=(-1,1) \subset \mathbb{R}$ and let $f$ be the function in Example 1.5. Define the function $g: \Omega \rightarrow \mathbb{R}$ as follows: $g(x):=f(x)$, for $x \in(-1,0]$ and $g(x)=1$ for $x \in(0,1)$. Then $g(x)$ is again quasi-continuous on $\Omega$ but now attains a weak local minimum at every point of $\Omega$. On the other hand we could define $h(x)=\frac{k-1}{k}$, for $x \in\left[\frac{1}{k+1}+\frac{1}{4 k+4 k^{2}}, \frac{1}{k}-\frac{1}{4 k+4 k^{2}}\right)$, and $h(x)=g(x)$ otherwise. Then $h(x)$ is constant on $[0,1)$ but again any open interval of $p_{0}=0$ in $\Omega$, must contain two different points $x_{1}, x_{2}$ such that $\left|h\left(x_{1}\right)\right|<\left|h\left(p_{0}\right)\right|<$ $\left|h\left(x_{2}\right)\right|$.

It is important to recall that functions which at every point attain weak local extremum certainly need not be quasi-continuous as the following example illustrates.

Example 1.6. Let $\Omega=(-1,1) \subset \mathbb{R}$ and define the function $f: \Omega \rightarrow \mathbb{R}$ by $f(x):=1$, for $x \in(-1,0) \cup(0,1)$, and $f(0):=0$. Clearly $f$ is Lebesgue measurable, locally bounded, but not quasi-continuous at the origin, which we may verify easily by the use of Proposition 1.3. However, $f$ certainly attains weak local extremum (in fact weak local minimum) at every point of $\Omega$. Indeed this is the case for any discrete lower semi-continuous function, and lower semicontinuity does not imply quasi-continuity. In fact, if we define $h(x)=0$ for $x \in(-1,0] \cup(1,2), h(x)=1$ for $x \in(0,1]$, we obtain a quasi-continuous function which is neither lower semi-continuous nor upper semi-continuous.

Sometimes we shall use the term discrete.

Definition 1.7. By a discrete subset of $\mathbb{R}^{n}$ we mean a subset such that every point is an isolated point in the topology of the ambient space and the set does not contain an accumulation point. In particular, for a bounded real-valued function, being discrete implies that the image is countable. It is known that for any uncountable subset $S \subset \mathbb{R}$ there are only a countable set of points 
of $S$ which are not accumulation points ${ }^{2}$ of $S$, thus any discrete set must be countable.

\section{NON-TRIVIALITY OF THE PROBLEM}

The situation is nontrivial already in one dimension and when the absolute value of a quasi-continuous function is finite.

Observation 2.1. Let $\Omega \subset \mathbb{R}$ be a bounded domain and $f: \Omega \rightarrow \mathbb{C}$ a bounded quasi-continuous function. In order for $f$ to attain weak local extremum at every point, it is in general, neither sufficient nor necessary that $|f|(\Omega)$ is finite.

Let us verify this observation by examples. Example 1.5 (specifically the function $g$ ) shows that even discreteness is not necessary (i.e. there are nondiscrete quasi-continuous functions with absolute value which has countable image, such that the functions attain weak local extremum at each point). For an example which illustrates non-sufficiency we have the following.

Example 2.2. Let $\Omega=(-1,1)$ and set,

$$
f(x)= \begin{cases}1 & , x \in(-1,0], \\ 0 & , x \in\left(\frac{1}{k+1}, \frac{2 k+1}{2 k(k+1)}\right], k \in \mathbb{Z}_{+}, \\ 2 & , x \in\left(\frac{2 k+1}{2 k(k+1)}, \frac{1}{k}\right], k \in \mathbb{Z}_{+},\end{cases}
$$

Then $f$ is quasi-continuous (one can for instance verify this by using Proposition 1.3) has finite image which in turn implies that $|f|(\Omega)$ is finite, but $x=0$ does not yield a a weak local extremum for $f$.

Here is a counter-example for higher dimension.

Example 2.3. Let $\Omega=\{(x, y):-1<x<1,-1<y<1\} \subset \mathbb{R}^{2}$ and define the function $f: \Omega \rightarrow \mathbb{R}$ as follows: $f(x, y):=0$ for $-1<x<0, f(x, y):=1$ for $0<x<1$ and $f(0, y)=I_{\mathbb{Q}}(y)$ where $I_{\mathbb{Q}}$ denotes the indicator function i.e. $I_{\mathbb{Q}}(y)=1$ if $y \in \mathbb{Q}$, and $I_{\mathbb{Q}}(y)=0$ otherwise. Then $f(x, y)$ is quasi-continuous on $\Omega$ (again this can be verified easily by the use of Proposition 1.3) and clearly the image $|f|(\Omega)=\{a \in \mathbb{R}: a=|f(x, y)|$, some $(x, y) \in \Omega\}=\{0,1\}$, in particular it is finite. However, for any open neighborhood, $U \subset \Omega$, of any point $\left(x_{0}, y_{0}\right)$ on the set $\Omega \cap\{x=0\}$, there exists at least four pairwise distinct points $y_{1}, \ldots, y_{4}$ (all distinct also from $y_{0}$ ) such that $\left\{y_{1}, y_{2}\right\} \subset \mathbb{Q} \cap U$ whereas

\footnotetext{
${ }^{2}$ See e.g. Munkres [9], p.194: Let $q_{1}, q_{2} \in S \backslash S_{A} q_{1} \neq q_{2}$, where $S_{A}$ denotes the set of accumulation points, $S_{A}$, of $S$. Then there exists open neighborhoods $V_{q_{j}} \ni q_{j}$ such that $\left\{q_{j}\right\}=V_{q_{j}} \cap S_{A}, j=1,2$, i.e. $V_{q_{1}} \cap S_{A} \neq V_{q_{2}} \cap S_{A}$. Hence, if $S \backslash S_{A}$ is uncountable then any basis for the topology must be uncountable. Since $\mathbb{R}$ is second countable we can conclude that the set of points in $S$ which are not accumulation points, must be at most countable. In fact it is known that given any uncountable $S \subset \mathbb{R}$ there is a countable subset $S^{\prime} \subset S$ such that for every $a \in S \backslash S^{\prime}$ and every $\epsilon>0$ both $(a-\epsilon, a) \cap S$ and $(a, a+\epsilon) \cap S$ are uncountable, see e.g. Bennett \& Lutzer [2], p.941.
} 
$\left\{y_{3}, y_{4}\right\} \subset U \cap \mathbb{R} \backslash \mathbb{Q}$. Hence, $f$ does not attain weak local extremum at any point belonging to the set $\Omega \cap\{x=0\}$.

Remark 2.4. One way to account for the underlying mechanism which causes the additional complication in Example 2.3, compared to Example 2.2, is that the restriction of a quasi-continuous function to a curve is not necessarily quasicontinuous. Clearly, the function $f(x, y)$ has restriction to the line segment $\Omega \cap\{x=0\}$, which is not quasi-continuous. In fact, it is not even sufficient that the quasi-continuous function be separately quasi-continuous (see Example 2.7).

Definition 2.5 (Separate quasi-continuity). Let $x=\left(x_{1}, \ldots, x_{n}\right) \in \mathbb{R}^{n}$ denote real-analytic coordinates centered at 0 . For a fixed constant $c=\left(c_{1}, \ldots, c_{n-1}\right) \in$ $\mathbb{R}^{n-1}$ we shall denote by $\widehat{x}_{c, j}$ the point $\left(c_{1}, \ldots, c_{j}, x_{j}, c_{j+1}, \ldots, c_{n-1}\right)$. Let $\Omega \subset$ $\mathbb{R}^{n}$. A function $f: \Omega \rightarrow \mathbb{C}$ is called separately quasi-continuous if for each $c \in \mathbb{R}^{n-1}$, the function $f\left(\widehat{x}_{c, j}\right)$ (where defined) is quasi-continuous on the intersection of $\Omega$ with the set of points $x \in \mathbb{R}^{n}$ such that $x_{k}=c_{k}, k \leq j$, and $x_{k}=c_{k+1}, k>j$.

Example 2.6. Let $\Omega_{j}, 1 \leq j \leq n$ be domains $\Omega_{j} \Subset \mathbb{R}$, Let $f_{j}\left(x_{j}\right)$ be quasicontinuous on $\Omega_{j}$ such that $\left|f_{j}\right|\left(\Omega_{j}\right)$ is discrete. Define $x=\left(x_{1}, \ldots, x_{n}\right)$, $\Omega:=\prod_{j=1}^{n} \Omega_{j} \subset \mathbb{R}^{n}$, and $f(x)=\prod_{j=1}^{n} f_{j}\left(x_{j}\right)$. Then clearly $|f|(\Omega)$ is quasicontinuous in each variable separately and $|f(x)|=\prod_{j}\left|f_{j}\left(x_{j}\right)\right|$, so the image of $|f|$ is discrete.

Quasi-continuity together with separate quasi-continuity such that the function separately attains weak local extremum at every point (i.e. the restrictions, with all but one variable fixed, attain weak local extremum at every point) does not imply that the function attains weak local extremum at every point.

Example 2.7. Let $\Omega=(-1,1) \times(-1,1) \subset \mathbb{R}^{2}$ and let $\left(x_{1}, x_{2}\right)$ denote Euclidean coordinates centered at $(0,0)$. Define $f\left(x_{1}, x_{2}\right)=2$ for $\Omega \cap\left\{x_{1}<0, x_{2}>\right.$ $0\}, f\left(x_{1}, x_{2}\right)=1$ for $\Omega \cap\left\{x_{2} \leq 0\right\}$, and $f\left(x_{1}, x_{2}\right)=0$ for $\Omega \cap\left\{x_{1} \geq 0, x_{2}>0\right\}$, Then $f$ is quasi-continuous on $\Omega$ and separately quasi-continuous in the sense of Definition 2.5. Clearly $|f|(\Omega)=\{0,1,2\}$ and the restrictions, with all but one variable fixed, attain weak local extremum at every point. However the point $(0,0)$ does not render a weak local extremum for $f$.

\section{Result}

In light of our examples we shall attempt to determine what can be said about the set of weak extrema given countability of the absolute value of a bounded quasi-continuous function, for arbitrary positive dimension $n$. We shall make use of the following.

Proof-tool 3.1. Let $f$ be a measurable complex-valued function on a domain $\Omega \subset \mathbb{R}^{n}$. Given a point $p_{0} \in \Omega$ and an $\epsilon>0$, we define,

$$
\omega_{p_{0}, \epsilon}^{f}:=\left\{z \in \Omega:\left|f(z)-f\left(p_{0}\right)\right|<\epsilon\right\}^{\circ},
$$


where we use the notation $X^{\circ}$ for the interior of a set $X$. Note that we always have $p_{0} \in\left\{z \in \Omega:\left|f(z)-f\left(p_{0}\right)\right|<\epsilon\right\}$ and that $\omega_{p_{0}, \epsilon}^{f}$ is always open but can be empty. It is known (see Daghighi [4]) that $f$ is quasi-continuous at a point $p_{0} \in \Omega$ iff if for any open ambient neighborhood $\mathrm{U}_{p_{0}}$ of $p_{0}, \omega_{p_{0}, \epsilon}^{f} \cap \mathrm{U}_{p_{0}} \neq \emptyset, \forall \epsilon>$ 0 .

Proposition 3.2. Let $\Omega \subset \mathbb{R}^{n}$ be a bounded domain and $f: \Omega \rightarrow \mathbb{C}$ a measurable, bounded, quasi-continuous function. Then the following holds true:

(i) $f$ attains weak local extremum at each point of a dense open subset of $\Omega$ whenever $|f|(\Omega)$ is countable.

(ii) If $f$ attains weak local extremum off a countable subset then $|f|(\Omega)$ is countable.

Proof. (i).

Define $\mathfrak{A}_{c}:=\{z \in \Omega:|f(z)|=c\}$ for $c \in \mathbb{R}_{\geq 0}$. By assumption there is a countable set $\left\{c_{j}\right\}_{j \in \mathbb{N}}$ such that, for any open subset $U \Subset \Omega$ we have,

$$
\bar{U}=\bigcup_{j \in \mathbb{N}}\left(\bar{U} \cap \mathfrak{A}_{c_{j}}\right) .
$$

We now show that this implies that at least one of the $\bar{U} \cap \mathfrak{A}_{c_{j}}$ contains an interior point: If at least one $\bar{U} \cap \mathfrak{A}_{c_{j}}$ is open we are already done. If instead all the sets (i.e. for each $j \in \mathbb{N}) \bar{U} \cap \mathfrak{A}_{c_{j}}$, are closed then we have a countable family of closed sets whose union $(=\bar{U})$ contains an interior point since $U$ is open. This implies (see e.g. Munkres [9], p.179) that at least one of the members of the cover must contain an interior point. This in turn implies that at least one of the sets $U \cap \mathfrak{A}_{c_{j}}$ has an interior point. Now, for any $\epsilon>0$, we are free to replace $U$ by the set $\omega_{p_{0}, \epsilon}^{f}$ (see Proof-tool 3.1). Furthermore, for any $\epsilon>0$, we can let $\mathrm{U}_{p_{0}}$ (appearing in Proof-tool 3.1) to be the ball, $B_{p_{0}}(\epsilon)$, in $\mathbb{R}^{n}$, centered at $p_{0}$ and of radius $\epsilon$. This implies that, for any small $\epsilon>0, B_{p_{0}}(\epsilon)$, must contain an interior point of some $\mathfrak{A}_{c_{j}}$. Finally, the set $\mathfrak{A}:=\bigcup_{j \in \mathbb{N}} \mathfrak{A}_{c_{j}}$ is open (as the union of open subsets) and it necessarily belongs to the set of weak local extrema of $f$ (any point, $q$, such that $|f|$ is constant on an open neighborhood of $q$ is necessarily both a weak local minimum and a weak local maximum). This proves (i).

(ii).

Let $\mathfrak{B}$ be the set of weak local extremum points of $f$. It is known, see e.g. Hirsch \& Lacombe [5], p.11, that the image of the set of weak local extrema of a realvalued function on a separable metric space, is countable. In particular $|f|(\mathfrak{B})$ is countable (and by assumption $\mathfrak{B}$ contains a dense open subset, $\mathfrak{A} \subset \Omega$ ). Let $\mathfrak{W}$ denote the set of isolated points of $\Omega \backslash \mathfrak{B}$, and,

$$
h(z):= \begin{cases}f(z) & , z \in \mathfrak{W} \\ 0 & , \text { otherwise. }\end{cases}
$$


Then, $h$ attains weak local extrema at each point of $\mathfrak{W}$, thus $|h|(\mathfrak{W})=|f|(\mathfrak{W})$ is countable. Hence the equality $|f|(\Omega)=|f|(\Omega \backslash \mathfrak{W}) \cup|f|(\mathfrak{W})$ completes the proof of (ii) and thereby also the proof of Proposition 3.2.

For a domain $\Omega \subset \mathbb{R}^{n}$, and an integrable function $f$ on $\Omega$, we denote by $[f]$, the $L^{1}(\Omega)$-equivalence class of $f$. Note that if a function is measurable and bounded on a bounded domain, then it is integrable on that domain. We also denote by $\operatorname{Hess}(\phi)$ the real Hessian (matrix) of $\phi \in C^{2}$.

Example 3.3. Let $\Omega \subset \mathbb{R}^{n}$ be a bounded domain and $f: \Omega \rightarrow \mathbb{C}$ a bounded, measurable, quasi-continuous function. Let $\phi \in C^{2}\left(\mathbb{R}^{n}, \mathbb{R}\right)$ be a function such that Hess $(f)$ is definite a.e. on $\Omega$ and assume the set of extreme points of $f$ is the intersection of $\Omega$ with $\{\phi<c\}$ for some constant $c \in \mathbb{R}$.

If $|f|(\Omega)$ is countable then $[g]=[f]$ for some $g$ attaining weak local extremum everywhere on $\Omega$.

This follows from the fact that the boundary of a strictly convex set has Lebesgue measure zero (see e.g. Lang [8], p.90). Let $p_{0} \in \Omega \cap\{\phi=c\}$ such that $\operatorname{Hess}(\phi)\left(p_{0}\right)$ is definite and assume w.l.o.g. $\operatorname{Hess}(\phi)\left(p_{0}\right)$ is positive definite (for negative definite we can repeat the arguments using the opposite orientation). This implies by continuity of the Hessian that there exists an open $U_{p_{0}} \subset \Omega \cap\{\phi=c\}$ such that $\operatorname{Hess}(\phi)$ on $U_{p_{0}}$, whence $U_{p_{0}}$ belongs to the boundary of a strictly convex subset of $\mathbb{R}^{n}$, so it has Lebesgue measure zero. Also the set of points where the Hessian is not definite are already of Lebesgue measure zero by the assumption on $\phi$ thus the set $\{\phi=c\} \cap \Omega$ is a subset of Lebesgue measure zero. On the other hand the conditions of the corollary imply that the set of extreme points, let us denote it by $\mathfrak{B}$, of $f$, coincide with $\{\phi<c\} \cap \Omega$. By Proposition 3.2, $\mathfrak{B}$ is dense in $\Omega$, thus the set $\Omega \backslash \mathfrak{B}=\partial \mathfrak{B} \cap \Omega$. (Note that $\Omega$ being open implies that $\partial \Omega \cap \partial \mathfrak{B}$ does not intersect $\Omega$ ). Therefore $\partial \mathfrak{B} \cap \Omega=\{\phi=c\} \cap \Omega$, is of Lebesgue measure zero (in $\mathbb{R}^{n}$ ). Hence, we can define,

$$
g(z)= \begin{cases}f(z) & , z \in \mathfrak{B} \\ 0 & , z \in \Omega \backslash \mathfrak{B}\end{cases}
$$

Then (because 0 is always a weak extreme value), $g$ attains weak local extremum at every point and $g=f$ except on a set of Lebesgue measure zero, i.e., $[f]=[g]$.

Since continuous functions are quasi-continuous we immediately have the following.

Example 3.4. Let $\Omega \subset \mathbb{R}^{n}$ be a bounded domain and $f: \Omega \rightarrow \mathbb{C}$ be a continuous function attaining weak local extremum at each point off a countable subset. Then $f$ must be of constant modulus on $\Omega$. 
Proof. By Proposition 3.2, the set $|f|(\Omega)$ is countable. Also $z \mapsto|f(z)|$ is continuous, thus $|f|(\Omega)$ must be connected, therefore $|f|$ reduces to a real constant on $\Omega$.

\section{REFERENCES}

1. E. Behrends, S. Geschke, T. Natkaniec, Functions for Which All Points Are Local Extrema, Real Anal. Exchange, 33 no.2 (2007), 467-470

2. H. Bennett, D. Lutzer, Subcompactness and Domain Representability in GO-spaces on Sets of Real Numbers, Topology and its Applications, 156, Iss. 5 (2009), 939-950

3. F.S. Cater, Functions with preassigned local maximum points, Rocky Mountain J. Math. 15, no.1 (1985), 215-218

4. A. Daghighi, Approach regions of lebesgue measurable, locally bounded, quasicontinuous functions, Int. Journal of Math. Analysis, 6 no.14 (2012), 659-680

5. F. Hirsch, G. Lacombe, Elements of Functional Analysis, GTM Springer 1993

6. S. Kempisty, Sur les fonctions quasicontinues, Fund. Math., 19 (1952), 184-197

7. S. Kasahara, Boundedness of Semicontinuous Finite Real Functions, Proc. Japan Acad., 33 (1957), 183-186

8. R. Lang, A note on measurability of convex sets, Arch.Math. 47, (1986), 90-92

9. J. Munkres, Topology, Second Edition, Pearson 2000

10. E.E. Posey, J.E. Vaughan, Functions with a proper local maximum in each interval, Amer. Math. Monthly 904 (1983), 281-282

11. A .Villani, Functions with a dense set of proper local maximum points, Proc. Amer. Math. Soc. 94 (1985), 353-359

\section{Received: May 5, 2014}

